\section{AB0368 PREDICTORS OF NEUROPATHIC PAIN IN RHEUMATOID ARTHRITIS}

T. Martins Rocha ${ }^{1}$, S. Pimenta ${ }^{1}$, M. Bernardes ${ }^{1}$, A. Bernardo ${ }^{1}$, M. Barbosa ${ }^{2}$, R. Lucas ${ }^{3}$, L. Costa ${ }^{1} .{ }^{1}$ Rheumatology; ${ }^{2}$ Pain Unit, Centro Hospitalar de São João; ${ }^{3}$ Clinical Epidemiology, Faculty of Medicine University of Porto (FMUP), Porto, Portugal

Background: Significant pain persists in a substantial proportion of Rheumatoid Arthritis (RA) patients in spite of disease remission. Recent evidence indicates that features suggestive of neuropathic pain (NP) are present in RA patients with a prevalence range of $33-38 \%$ using the painDETECT questionnaire (PDQ). Objectives: To estimate the clinical predictors of NP in a cohort of RA patients. Methods: Observational, cross-sectional study was performed with RA patients followed at our Rheumatology department with unchanged DMARD treatment during the last 3 months. Patients with diagnosed neuropathy or non-RA risk factors for NP were excluded. Selected patients were evaluated in a medical visit. Demographic, clinical and laboratorial data were collected and disease activity and functional measures were evaluated. Two questionnaires were applied to assess NP: the Leeds Assessment of Neuropathic Symptoms (LANSS) and the $\mathrm{PDQ}$. Univariate and multivariate logistic regression were performed to identify the predictors of NP. Significance level was set as $<0.05$.

Results: 112 RA patients were included. $86(77 \%)$ were females, with a mean (SD) age of 55.1 (10.8) years and median disease duration of 13 years (range: 2-41). $84 \%$ patients were seropositive for Rheumatoid Factor and/or ACPA. 102 $(91 \%)$ were treated with DMARDs and $42 \%$ with a biologic DMARD, whom $8 \%$ in monotherapy. The mean (SD) DAS28 4V was $3.61(1.01)$ and $12 \%$ were in remission. $45(40 \%)$ patients had NP by the LANSS $(\geq 12), 28 \%$ had a possible/likely NP in the PDQ $(>12)$ and $21 \%$ were positive in the both tests. Female sex was predictive of LANSS and PDQ NP (OR: 3.44, $p=0.02$ and OR: 3.70; $p=0.05$, respectively) and disease duration was a predictor of LANSS (OR: 0.92 per year, $p=0.004$ ). After adjusting for these variables, pain VAS, patient global activity and the tender joint count were positive predictors of NP by both tests. Swollen joint count, ESR or CRP levels were not significantly associated with NP. DAS28 CRP and HAQ scores were both significant positive predictors of PDQ NP (OR: 1.77 and OR: 3.61, $\mathrm{p}<0.01$, respectively). DAS28 CRP, dichotomized as remission/non-remission, and the HAQ score were also significantly positive predictors of LANSS NP (OR: 5.55, $p=0.01$ (DAS28 CRP $\geq 2.6$ ); OR: 2.15, $\mathrm{p}=0.03$ ). Positivity for ACPA was a negative predictor of LANSS NP (OR: $0.23, p=0.009$ ), remaining significant after adjusting for DAS28 CRP, HAQ and current methotrexate (MTX). Number of analgesics and current NSAIDs treatment were associated with PDQ NP (OR: 2.72 and OR: 3.40, respectively, $\mathrm{p}<0.05$ ). Current MTX and previous/current Hydroxychloroquine (HCQ) treatment were both negative predictors for PDQ NP (OR: 0.41 and OR: 0.20 , respectively, $\mathrm{p}<0.05$ ). No other significant associations were found. In a subgroup with dosed TSH levels $(n=18)$, there was a positive though non-significant association with LANSS and PDQ (OR: 3.12, OR: 14.98, $p=0.09$ ).

Conclusions: NP was present in sizable proportion of RA patients. Consistently with previous data, our study supports the association between NP and disease activity/functional scores but not with objective inflammatory measures. This study newly points to a possible protective role of ACPA positivity, MTX and HCQ treatment in NP risk.

References:

[1] Koop SM, et al. Arthritis Res Ther. 2015; 17:237.

[2] AW Christensen et al. Scand J Rheumatol 2016;1-9.

Disclosure of Interest: T. Martins Rocha Grant/research support from: Portuguese Society of Rheumatology/Alfa Wassermann on May 2015, S. Pimenta: None declared, M. Bernardes: None declared, A. Bernardo: None declared, M. Barbosa: None declared, R. Lucas: None declared, L. Costa: None declared DOI: 10.1136/annrheumdis-2017-eular.2634

\section{AB0369 VITAMIN D STATUS AND DISEASE ACTIVITY IN PATIENTS WITH RHEUMATOID ARTHRITIS}

V. Povoroznyuk ${ }^{1}$, O. Synenky ${ }^{2}$, N. Balatska ${ }^{1} .{ }^{1}$ D.F. Chebotarev Institute of Gerontology Nams Ukraine, Kyiv; ${ }^{2}$ Lviv Regional Clinical Hospital, Lviv, Ukraine

Background: The purpose of the study was to define the associations between serum level of 25 -hydroxyvitamin $\mathrm{D}(25(\mathrm{OH}) \mathrm{D})$ and disease activity in patients with rheumatoid arthritis (RA).

Objectives: The study group comprised 93 patients aged 27-80 years suffering from RA (74.2\% women) and 93 controls.

Methods: The levels of rheumatoid factor (RF) and C-reactive protein (CRP) were measured by immunoturbidimetric assay. Erythrocyte sedimentation rate (ESR) was measured by Westergren method. Serum $25(\mathrm{OH}) \mathrm{D}_{\text {total }}$ level was determined by electrochemiluminescence $(E C L)$ method. The optimal vitamin $D$ status was defined when serum $25(\mathrm{OH})$ D level was $30-50 \mathrm{ng} / \mathrm{ml}$.

Results: In general, $54.8 \%$ of RA patients revealed vitamin $D$ deficiency, and $37.6 \%$ - vitamin $D$ insufficiency. No significant difference was found when mean serum 25(OH)D levels in RA patients and healthy controls where compared. 25(OH)D levels appeared markedly lower in patients with the highest activity of RA compared to values noted in patients with the minimal RA activity $(16.55 \pm 9.26$ vs $22.59 \pm 9.74 \mathrm{ng} / \mathrm{ml}, \mathrm{p}<0.05)$. In RA patients $25(\mathrm{OH}) \mathrm{D}$ levels were significantly and negatively associated with markers of disease activity (DAS28-ESR $(\beta=$
$-0.33 ; 95 \% \mathrm{Cl}=0.05,-0.01), \mathrm{CRP}(\beta=-0.23 ; 95 \% \mathrm{Cl}=-0.72 ; 0.00)$, and $\mathrm{ESR}$ $(\beta=-0.26 ; 95 \% \mathrm{Cl}=-0.78 ;-0.10)$. All these associations remained statistically significant after adjustment for gender, age and BMI.

Conclusions: Vitamin $\mathrm{D}$ deficiency should be considered as an important predictor of the high RA activity (AUROC $=0.67 ; 95 \% \mathrm{Cl}=0.513-0.83, \mathrm{P}=0.05$ ).

Disclosure of Interest: None declared

DOI: 10.1136/annrheumdis-2017-eular.4600

\section{AB0370 DEVELOPMENT OF PATIENT PREFERENCE PHENOTYPES FOR RHEUMATOID ARTHRITIS}

W.B. Nowell ${ }^{1}$, C. Wiedmeyer ${ }^{1}$, G. Herath ${ }^{2}$, G. Michel ${ }^{3}$, L. Fraenkel ${ }^{2} .{ }^{1}$ Global Healthy Living Foundation, Upper Nyack; ${ }^{2}$ Yale University School of Medicine;

${ }^{3}$ Yale University, New Haven, CT, United States

Background: Many important treatment decisions for patients with rheumatoid arthritis (RA) are conditional on patient preferences and, according to the U.S. National Academy of Medicine, mandate a shared decision making approach (SDM). Furthermore, SDM is being increasingly recognized as an important quality measure. One of the most common preference sensitive decisions in RA is how to escalate care when response to methotrexate monotherapy is inadequate. However, the number of RA medications currently approved makes it challenging for patients to weigh the pros and cons related to each of the treatment options in order to develop a preference.

Objectives: The objective of this study was to develop representative patient preference phenotypes to enable patient-physician dyads to effectively incorporate patient preferences at the point-of-care.

Methods: People living with RA were invited to complete a Choice-Based Conjoint analysis survey including seven attributes (route of administration, time to onset of action, bothersome adverse events (AEs), serious AEs, extremely rare AEs, duration of time on the market and affordability) developed iteratively based on patient feedback. Each attribute was described across three or four levels using plain language. Preference phenotypes were identified by applying latent class analysis to the conjoint data. Class solutions were replicated five times from random starting seeds. A five-group solution was chosen based on Akaike's information criterion. We calculated the percentage of importance assigned to each attribute and performed simulations to estimate preferences for triple therapy, SC and IV biologics, or tofacitinib.

Results: 1100 U.S. subjects recruited via the CreakyJoints online patient community completed the survey. Of these, 49 were eliminated because they completed the survey in less than 10 minutes and an additional 45 people were excluded because they did not respond correctly to a dominant choice task. The mean age was 51.7 (11.2). The majority were female; $(92 \%)$ and Caucasian (93\%). Preferences (assuming low cost across options), and the reasons underlying each respondent's preference, clustered into five groups (Figure 1). There were no differences in the distribution of demographic or clinical characteristics across the five groups. Phenotypes were created based on the stated preference data.

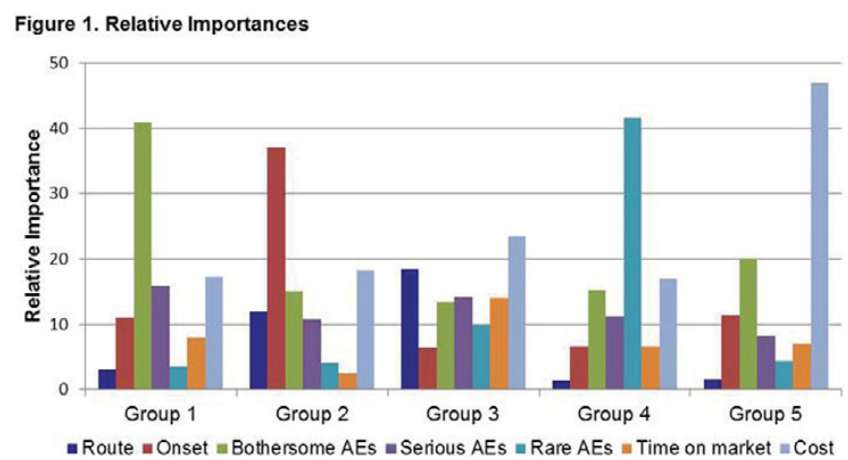

Conclusions: RA patients' preferences vary and can be classified into distinct phenotypes. Ongoing research is evaluating whether enabling patients to identify with a preference phenotype facilitates SDM at the point-of-care.

Acknowledgements: This research was supported by a grant from the Rheumatology Research Foundation.

Disclosure of Interest: None declared

DOI: 10.1136/annrheumdis-2017-eular.5024

\section{AB0371 THE QUALITY OF LIFE CAN BE IMPROVED BY FOREFOOT RECONSTRUCTION EVEN IN PATIENTS WITH WELL-CONTROLLED RHEUMATOID ARTHRITIS}

Y. Nomura ${ }^{1}$, H. Ishikawa ${ }^{1}$, A. Abe ${ }^{1}$, S. Ito ${ }^{1}$, T. Kojima ${ }^{2}$, M. Kojima ${ }^{3}$

N. Ishiguro ${ }^{2}$, A. Murasawa ${ }^{1}{ }^{1}$ Rheumatology, Niigata Rheumtic Center, Shibata; ${ }^{2}$ Orthopedic Surgery, Nagoya University Hospital; ${ }^{3}$ Medical Education, Nagoya City University Hospital Graduate School of Medicine, Nagoya, Japan

Background: The treatment aim of rheumatoid arthritis (RA) is achieving and 